\title{
28 Research Soure \\ Efficacy of Apatinib Combined with FOLFIRI in the First-Line Treatment of Patients with Metastatic Colorectal Cancer
}

\section{Xuetong Rong}

Shanxi Medical University

Haiyi Liu

Affiliated Center Hospital of ShanXi Medical University

Hongmei Yu

Shanxi Medical University

Jian Zhao

Affiliated Center Hospital of ShanXi Medical University

Jie Wang

Shanxi Medical University

Yusheng Wang ( $\nabla$ wangyusheng1972@163.com )

Affiliated Center Hospital of ShanXi Medical University

\section{Research Article}

Keywords: Apatinib, FOLFIRI, Colorectal adenocarcinoma, Survival

Posted Date: December 8th, 2021

DOI: https://doi.org/10.21203/rs.3.rs-833709/v1

License: () (1) This work is licensed under a Creative Commons Attribution 4.0 International License.

Read Full License

Version of Record: A version of this preprint was published at Investigational New Drugs on January 8th, 2022. See the published version at https://doi.org/10.1007/s10637-021-01205-3. 


\section{Abstract}

Objective: To evaluate the efficacy and safety of apatinib combined with FOLFIRI in the first-line treatment of advanced metastatic colorectal cancer (mCRC) and explore potential factors of efficacy.

Methods: Twenty mCRC patients treated at Affiliated Cancer Hospital of Shanxi Medical University from March 2017 to March 2019 were included according to the enrolment criteria. They provided informed consent and were treated with apatinib combined with FOLFIRI according to the scheduled regimen until disease progression or unacceptable toxicity occurred. The primary endpoint was OS. The secondary endpoints included PFS, ORR, DCRand safety. OS and PFS were calculated using Kaplan-Meier curves. Univariate and multivariate Cox regression analyses were used to evaluate independent prognostic factors of OS and PFS. R was used to determine cut-off values for biochemical indicators. Forest maps were drawn for Cox univariate results and the relationships between NLR and ECOG, which were significant in univariate analysis, and OS were represented by Kaplan-Meier curves.

Results: The median OS and PFS were 16.135 months (95\% Cl: $9.211-22.929)$ and 6 months (95\% Cl: 5.425-6.525). Multivariate Cox analysis showed that NLR and CEA were independent prognostic factors. The most common grade 3-4 adverse events were hypertension, diarrhoea, increased alkaline phosphatase, decreased leukocytes and decreased neutrophils.

Conclusion: Apatinib combined with FOLFIRI for the first-line treatment of advanced unresectable mCRC showed good efficacy and safety. The baseline NLR was predictive of efficacy, and a low baseline NLR (HR: 0.2895, $P=0.0084$ ) was associated with improved OS.

\section{Introduction}

The incidence and mortality of colorectal cancer (CRC) rank third among all malignant tumours worldwide [1]. In China, CRC is the fifth most common malignant tumour [2]. The early diagnosis rate of CRC in China is low. CRC is often in the late stage when diagnosed, with no chance of surgical resection and a short survival period, which seriously threatens the health of people. The treatment of advanced unresectable CRC is mainly systemic treatment. In recent decades, the progress of chemotherapy has promoted the survival of patients with advanced metastatic CRC. Oxaliplatin combined with the 5-FU/ Calcium Folinate regimen (FOLFOX) and irinotecan combined with the 5-FU/ Calcium Folinate regimen (FOLFIRI) are the basic regimens for advanced CRC [3]. The V308 study [4] mentioned that FOLFIRI was approved as the first-line treatment for advanced metastatic CRC by the European and US Food and Drug Administration (FDA) in 2012 [5], and FOLFIRI was also the first recommendation in the Chinese Society of Clinical Oncology (CSCO) guidelines. However, the median survival time of systemic chemotherapy alone was less than 27 months [6]. The advent of targeted drugs has pushed the treatment of advanced $\mathrm{CRC}$ to a new stage and extended the survival time of patients. Anti-VEGF and anti-EGFR monoclonal antibodies are currently recognized as targeted therapeutic options for advanced CRC [7], but the antiEGFR monoclonal antibodies panitumumab and cetuximab have significant effects on patients with wild- 
type RAS and BRAF, while they have poor effects on patients with mutant RAS and BRAF. Although bevacizumab, an anti-VEGF monoclonal antibody, is effective in the overall population of CRC patients, the toxicities of bleeding, perforation and obstruction cannot be ignored, especially in patients with ulcerative primary lesions, who tend to have severe adverse reactions. Therefore, it is necessary to explore new therapeutic modes to improve the survival of patients with advanced CRC. Apatinib tablet is a smallmolecule targeted drug against VEGFR-2 that blocks downstream signal transduction and inhibits tyrosine kinase production through highly selective competition for ATP binding sites of intracellular

receptor-2, thus inhibiting neoangiogenesis in tumour tissues and finally achieving the purpose of tumour treatment [8]. Apatinib has a therapeutic effect on various types of cancers. Current exploratory studies of apatinib in advanced CRC focus on later-line treatments. The efficacy of apatinib in first-line treatment is not yet clear. The objective of this open exploratory clinical trial was to evaluate the efficacy and safety of apatinib in combination with FOLFIRI in patients with advanced unresectable metastatic CRC and to explore clinical factors associated with prognosis.

\section{Materials And Methods}

\section{Ethics}

This is a single-centre, single-arm open exploratory clinical trial that has been registered in the China Clinical Research Network (ChiCTR1800015308) and approved by the Ethics Committee of Shanxi Cancer Hospital under careful review (Approval number: 201719).

\section{Patients and grouping}

From March 2017 to March 2019, all patients received treatment at the Affiliated Cancer Hospital of Shanxi Medical University. The inclusion criteria were as follows. Patients were aged $18-75$ years, and there were no limitations on sex. Patients were diagnosed with colorectal adenocarcinoma with concurrent distant metastasis by pathology or patients had undergone radical resection of their primary tumour. According to Response Evaluation Criteria in Solid Tumors (RECIST) 1.1, patients had at least one measurable target lesion (tumour lesion $10 \mathrm{~mm}$ long diameter on computed tomography (CT) scan, lymph node lesion $15 \mathrm{~mm}$ short diameter on CT scan, scan thickness no greater than $5 \mathrm{~mm}$, and no local treatment). Patients had an expected survival time of 3 months. Patients had an Eastern Cooperative Oncology Group (ECOG) physical condition score of $0-2$. The function of major organs was good; that is, the relevant examination indexes within 14 days before enrolment met the following requirements: A. routine blood examination: haemoglobin $90 \mathrm{~g} / \mathrm{L}$; neutrophil count $1.510^{\wedge} \mathrm{g} / \mathrm{L}$; platelet count 100 10^9/L; and white blood cell count 3.5 10^9/L; B. biochemical examination: total bilirubin 1.5 upper limit of normal (ULN); alanine aminotransferase (ALT) or aspartate aminotransferase (AST) 2.5 ULN; ALT or AST 5 ULN if liver metastases;serum creatinine (Cr) $1 \mathrm{ULN}$, endogenous $\mathrm{Cr}$ clearance rate $50 \mathrm{~mL} / \mathrm{mins}$ (Cockcroft-Gault formula); women of childbearing age must have a negative pregnancy test (serum or urine) within 7 days prior to enrolment and voluntarily use an appropriate method of contraception during 
observation and 8 weeks after the last administration of apatinib mesylate tablets. The subjects voluntarily joined the study and signed the informed consent form.

\section{Treatment regimen}

All subjects received the first-line treatment regimen of apatinib combined with

FOLFIRI: apatinib tablet $250 \mathrm{mg}$ once a day, orally with warm water half an hour after a meal (the time the drug is taken should be the same as much as possible). After one week, if the adverse reactions were well tolerated, the dose was adjusted to $500 \mathrm{mg}$ once a day orally. In the case of grade III adverse reactions, the dose could be reduced to $250 \mathrm{mg}$ once a day. Treatment discontinuities due to adverse events were permitted for no more than 14 days. Subjects were treated until disease progression or death from unacceptable toxicity, and the apatinib FOLFIRI regimen was as follows: irinotecan 180 $\mathrm{mg} / \mathrm{m}^{2}$ continued intravenously for $90 \mathrm{mins}$, day 1 ; calcium tetrahydrofolate $400 \mathrm{mg} / \mathrm{m}^{2}$, intravenous infusion ( $2 \mathrm{~h}$ ), day 1 ; fluorouracil $0.4 \mathrm{~g} / \mathrm{m}^{2}$ intravenously ( $1 \mathrm{~h}$ after calcium tetrahydrofolate); fluorouracil $2.4 \mathrm{~g} / \mathrm{m}^{2}$, continuous intravenous infusion (chemotherapy pump infusion) for $46 \mathrm{~h}$, repeated every 14 days.

\section{Data collection}

Before treatment, routine blood biochemical indicators, such as coagulation function indicators, were collected, and the neutrophil-to-lymphocyte ratio (NLR) in routine blood was calculated as the absolute count of neutrophils divided by the absolute count of lymphocytes. All subjects underwent CT 1 cycle after baseline, every 2 cycles thereafter and at disease progression. Overall survival (OS) was defined as the time from randomization to death from any cause. Progression-free survival (PFS) was defined as the time from randomization to tumour progression or death. Subjects must have a measurable tumour focus at baseline. Efficacy was assessed as complete response (CR), partial response (PR), stable disease (SD), and progressive disease (PD) according to RECIST 1.1. The objective response rate (ORR) is the proportion of patients whose tumours shrank to a certain extent and remained so for a certain period of time, including those with CR and PR. The disease control rate (DCR) was defined as the proportion of patients with CR, PR, and SD. Adverse events were graded from 0 to 4 based on the National Cancer Institute Common Terminology Criteria for Adverse Events (NCI-CTCAE 4.0).

\section{Statistical analysis}

The baseline demographics and disease characteristics were treated as categorical variables. R 3.4.4 software was used to determine thresholds for the baseline NLR, carbohydrate antigen 19-9 (CA19-9) and carcinoembryonic antigen (CEA) levels through the survivalROC package. The Kaplan-Meier (KM) method was used to analyse OS and PFS. A Cox proportional hazards regression model was used for univariate and multivariate analyses, the univariate analysis results were displayed in a forest map, and the variables with statistical significance in the univariate analysis were plotted with KM curves. The results included curves of the relationship between NLR and OS and the relationship between ECOG and OS. A variable with $P$ value $<0.20$ in univariate analysis was set as a covariable, and $P$-value $<0.05$ was 
considered statistically significant in the multivariate analysis. Finally, the statistically significant results are presented in a table. All statistical estimates were two-sided, and P-value $<0.05$ was considered statistically significant. SPSS version 26.0 software and RStudio were used for the analyses, and the $\mathrm{R}$ packages used included forestplot, grid, magrittr, and checkmate.

\section{Results}

\section{Patient characteristics}

From March 2017 to March 2019, 40 patients were screened, and a total of 20 subjects met the inclusion criteria (Figure 1). As of June 1, 2021, the median follow-up time was 21.9 months (7.73 months-36.23 months). Among the 20 registered subjects, 20 (100\%) withdrew from the study due to disease progression, of which 15 died (75\%) and 5 (25\%) had drug-related adverse events. The baseline demographics and pretreatment characteristics are shown in Table 1. The median age was 59.5 years (44-71 years), and approximately half of the subjects were male (12[60\%]). About one-third of the subjects had an ECOG performance status score of 2. Most subjects had multiple sites of metastasis (15 $[75 \%])$, and the liver and lung were the most common sites of metastasis $(20[100 \%])$. None of the subjects had received any antitumour treatment before enrolment.

\section{Efficacy analysis}

Seven subjects (35\%) achieved PR, nine subjects (45\%) achieved SD, and three subjects (15\%) developed PD. The results of the efficacy analysis are shown in Figure 2. One subject did not undergo CT examination during the second treatment cycle, so an efficacy evaluation could not be performed.

Table.1 Patients characteristics 


\begin{tabular}{|c|c|}
\hline Patient characteristic & Value (n. \%) \\
\hline Median age, years (range) & $59.5(44-71)$ \\
\hline \multicolumn{2}{|l|}{ Sex } \\
\hline Male & $12(60)$ \\
\hline Female & $8(40)$ \\
\hline \multicolumn{2}{|l|}{ ECOG performance status } \\
\hline 1 & $14(70)$ \\
\hline 2 & $6(30)$ \\
\hline \multicolumn{2}{|l|}{ Primary tumour location } \\
\hline Left & $1(5)$ \\
\hline Right & $5(25)$ \\
\hline Rectum & $11(55)$ \\
\hline Sigmoid colon & $3(15)$ \\
\hline \multicolumn{2}{|l|}{ Differentiation } \\
\hline Moderate & $14(70)$ \\
\hline Low & $6(30)$ \\
\hline \multicolumn{2}{|l|}{ KRAS mutation } \\
\hline No & $5(25)$ \\
\hline Yes & $5(25)$ \\
\hline Unknown & $10(50)$ \\
\hline \multicolumn{2}{|l|}{ Metastatic site } \\
\hline Lung & $11(55)$ \\
\hline Liver & $15(75)$ \\
\hline Multi-site & $14(70)$ \\
\hline \multicolumn{2}{|l|}{ NLR } \\
\hline$<3.855$ & $12(60)$ \\
\hline$\geq 3.855$ & $8(40)$ \\
\hline \multicolumn{2}{|l|}{ CA199 } \\
\hline$<428.735$ & $17(85)$ \\
\hline
\end{tabular}




\begin{tabular}{ll}
$\geq 428.735$ & $3(15)$ \\
\hline CEA & \\
\hline$<39.645$ & $13(65)$ \\
$\geq 39.645$ & $7(35)$ \\
\hline Surgery & \\
\hline Radical mastectomy & $6(30)$ \\
\hline Palliative surgery & $8(40)$ \\
\hline None & $6(30)$
\end{tabular}

Table.1 Patients' baseline characteristics $(\mathrm{N}=20)$. ECOG, Eastern Cooperative Oncology Group; NLR, neutrophil-to-lymphocyte ratio; $\mathrm{LDH}$, lactate dehydrogenase; CEA, carcinoembryonic antigen; CA19-9, carbohydrate antigen 19-9

\section{Survival analysis}

At the data cut-off, all enrolled subjects had completed treatment and were available for survival analysis. The median PFS and OS were 6 (95\% confidence interval (Cl) 5.475-6.525) and 16.135 (95\% Cl 9.21122.929) months, respectively (Figure 3 ).

\section{Safety analysis}

The overall adverse event rate was $100 \%$ in the 20 subjects, but the majority of patients experienced grade 1 or 2 toxicities; $50 \%$ of the patients experienced hypertension, nausea, fatigue, hand-foot skin reaction, or leukopenia; and 6 (30\%) subjects had grade 3-4 adverse events, including hypertension, diarrhoea, alkaline phosphatase increase, leukopenia, and neutropenia. The adverse events are shown in Table 2.

Table. 2 Adverse events 


\begin{tabular}{llll} 
Adverse event & Grade 1-2 (n,\%) & Grade 3-4 (n,\%) & Total(n,\%) \\
\hline Nonhaematologic & & & \\
\hline Hypertension & $16(80)$ & $2(10)$ & $18(90)$ \\
\hline Vomiting & $18(90)$ & 0 & $18(90)$ \\
\hline Fatigue & $13(65)$ & 0 & $13(65)$ \\
\hline Hand-foot syndrome & $13(65)$ & 0 & $13(65)$ \\
\hline Abdominal pain & $4(20)$ & 0 & $4(20)$ \\
\hline Diarrhoea & $2(10)$ & $1(5)$ & $3(15)$ \\
\hline Bleeding & $4(20)$ & 0 & $4(20)$ \\
\hline Mucositis oral & $6(30)$ & 0 & $6(30)$ \\
\hline Proteinuria & $7(35)$ & 0 & $7(35)$ \\
\hline Serum AST increased & $5(25)$ & 0 & $5(25)$ \\
\hline ALP increased & $1(5)$ & $1(5)$ & $2(10)$ \\
\hline Hyperbilirubinemia & $9(45)$ & 0 & $9(45)$ \\
\hline Haematologic & & $1(5)$ & $9(45)$ \\
\hline Leukopenia & $15(75)$ & $1(5)$ & $7(35)$ \\
\hline Neutropenia & $8(40)$ & 0 & \\
\hline Thrombocytopenia & $7(35)$ & & \\
\hline
\end{tabular}

Table.2 Adverse events in the whole cohort $(n=20)$

\section{Exploratory analysis of molecular markers}

The relationship between clinical outcomes and several variables (including baseline characteristic laboratory parameters and drug-related adverse events) was analysed in the enrolled subjects. Receiver operating characteristic (ROC) curves were used to determine critical values. The threshold for NLR was 3.855 to distinguish between low (NLR \& LT 3.855) and high (NLR 3.855) subjects, the threshold for CA19-9 was 428.735 to distinguish between low (NLR \& LT 428.735) and high (NLR 428.735) subjects, and the threshold for CEA was 39.645 to distinguish between low (NLR \& LT 39.645) and high (39.645) subjects. The area under the curve (AUC) for NLR was 0.7867, the AUC for CA19-9 was 0.6 , and the AUC for CEA was 0.5467. Subject characteristics, including age, sex, tumour location, degree of differentiation, KRAS gene mutation status, number of metastatic sites, metastatic sites and CA19-9, were independent predictors of PFS and OS (Figure 4). The relationship between ECOG and OS ( $P=0.032)$ and the relationship between NLR and OS $(P=0.013)$, which were significant in the univariate analysis, were 
visualized by the KM method (Figure 5). In the multivariate Cox regression analysis, low baseline NLR (hazard ratio (HR): 9.767, 95\% Cl: 1.333-71.554, $\mathrm{P}=0.025)$ and low baseline CEA (HR: $17.691,95 \% \mathrm{Cl}$ : 1.163-269.081, $P=0.039$ ) were significantly correlated with increased OS, as shown in Table 3 . The results of multivariate analysis showed that NLR and CEA are independent prognostic factors of OS.

Table.3 Multivariate analysis

\begin{tabular}{|lllll|}
\hline Variable & PFS & \multicolumn{2}{c|}{ OS } & \\
& HR $(95 \% \mathrm{Cl})$ & P value & HR $(95 \% \mathrm{Cl})$ & P value \\
\hline NLR & & & & \\
\hline$<3.85 / \geqq 3.855$ & $3.115(0.533-18.195)$ & 0.207 & $9.767(1.333-71.554)$ & 0.025 \\
\hline CEA & & & & \\
\hline$<39.645 / \geqq 39.645$ & $5.824(0.518-65.419)$ & 0.153 & $17.691(1.163-269.081)$ & 0.039 \\
& & & & \\
\hline
\end{tabular}

Table.3 Multivariate analysis of factors to predict progression-free survival and overall survival of apatinib. PFS, progression-free survival; OS, overall survival; NLR, neutrophil-to-lymphocyte ratio; CEA, carcinoembryonic antigen; $\mathrm{HR}$, hazard ratio

\section{Discussion}

The incidence of CRC ranks third among all malignant tumours, and it is the fourth most deadly cancer worldwide [9]. The incidence and mortality of CRC in Asia have shown upward trends [10]. The early diagnosis rate of CRC in mainland China is low, and the disease is often in the late stage when diagnosed. At that point, the opportunity for surgical resection has been lost, the survival rate is low, and the mortality rate is high. Targeted therapy combined with chemotherapy has significantly improved the survival of patients with advanced CRC [11]. The targeted drugs for CRC that have been approved in China are bevacizumab and cetuximab. Cetuximab is mainly used for KRAS, NRAS, and BRAF wild-type CRC. Bevacizumab has obvious adverse reactions when applied to patients with bleeding, obstruction, and perforation tendencies, so many factors should be considered in the clinic when deciding which targeted drugs should be used. FOLFOX and FOLFIRI are commonly used options for metastatic CRC, and the two are used equivalently in first-line treatment. The two chemotherapy regimens have their own adverse reaction spectrum and are applied to different groups of people. Targeted drugs combined with chemotherapy regimens are currently the main means for treating advanced CRC. The selection of suitable targeted drugs and chemotherapy regimens is the main factor affecting the survival of advanced CRC patients. There are still many patients in the clinic who cannot be treated with the above two targeted drugs due to various factors, so more treatment methods need to be explored. 
Apatinib is a small molecule inhibitor that specifically binds to vascular endothelial growth factor receptor 2 (VEGFR-2). Blocking VEGFR-2 can inhibit the migration and proliferation of vascular endothelial cells and reduce the microvessel density of tumours, thereby inhibiting tumour angiogenesis and exerting antitumour effects [12,13]. Apatinib can enhance endoplasmic reticulum autophagy by enhancing the stress of oestrogen receptors to promote proteasome degradation, thereby inducing the apoptosis of CRC cells [14]. At the same time, apatinib inhibits the AKT-mTOR signalling pathway and increases the expression of Lc3-II to induce autophagy. In the process of autophagy, the UIK1 autophagy complex can be positively regulated by inhibiting $\mathrm{mTORC} 1$, thereby promoting autophagy and apoptosis [15]. Studies have shown that apatinib increases the ubiquitination of $\beta$-catenin and reduces the phosphorylation of Ser-9 in GSK3 $\beta$, thereby reducing the nuclear localization of $\beta$-catenin and inhibiting the VEGFR2- $\beta$-catenin pathway to exert an antitumour effect [16]. Studies have shown that apatinib monotherapy for the late-line treatment of patients with advanced metastatic CRC who have failed chemotherapy has shown a good clinical effect compared with the control. The median PFS was 2 months, and the OS was 5 months longer than that of the control observation group. It is believed that apatinib has a certain clinical effect on the improvement of OS [17]. Therefore, we designed the regimen of apatinib combined with FOLFIRI to explore the efficacy and safety of this regimen as first-line therapy for advanced metastatic CRC.

In our study, the median OS of apatinib combined with FOLFIRI in the treatment of advanced unresectable CRC was 16.135 months (95\% Cl: 9.211-22.929), and the median PFS was 6 months (95\% Cl: $5.475-6.525)$. The ORR was $35 \%$, and the DCR was $80 \%$. The common adverse events were hypertension (90\%), vomiting (90\%), fatigue (65\%), hand-foot syndrome (65\%), white blood cell reduction ( $80 \%)$, neutropenia (45\%), and hyperbilirubinemia (45\%). Serious adverse events (grade $>3$ ) were hypertension (11.1\%), diarrhoea (5\%), increased ALP (5\%), leukopenia (5\%), and neutropenia (5\%). This study shows that the combination of apatinib and FOLFIRI as a first-line treatment for advanced unresectable metastatic CRC has good efficacy and tolerable adverse reactions. Compared with the previous treatment data of regorafenib, the first-line application of an apatinib combination regimen can give patients a better benefit. In the global CORRECT [18] study, regorafenib was used as a third-line targeted drug to treat advanced metastatic CRC in subjects who were frail or unsuitable for treatment. The median PFS was 1.9 months, and the OS was 6.4 months, which were improved compared to those of previous placebo treatment and best supportive treatment. In the CONCUR study, the median PFS and OS of Asians using regorafenib were 3.2 months and 8.8 months, respectively [19]. In clinical studies of regorafenib as a first-line application, the median PFS was 5.6 months, and the median OS was 16 months [20]. Our results are better than the first-line treatment data of single-agent regorafenib. The subjects we enrolled had the following characteristics: all subjects were stage IV patients at the time of enrolment, 14 (70\%) subjects had more than two metastatic sites, and of $15(75 \%)$ subjects with simultaneous liver metastases, 11 (55\%) subjects had lung metastasis, and 6 (30\%) subjects had an ECOG performance status score of 2 points. The liver is the most common site of metastasis in patients with advanced CRC. Approximately half of CRC patients will develop liver metastasis because most of the mesentery drains into the hepatic portal vein system [21], and liver 
invasion and metastasis can lead to poor prognosis [twenty-two]. The lobe of the lung is the second most common metastatic site of CRC after the liver [23], and lung metastasis develops more slowly than liver metastasis. This study included many patients with multisite metastases. At the time of enrolment, the subjects had a poor physical condition and poor tolerance, so the prognosis was poor. However, after the administration of apatinib combined with the FOLFIRI regimen, a certain curative effect was achieved, and there were tolerable adverse reactions.

In our study, the subjects' OS varied in length, with the longest being 26.7 months and the shortest being 4.47 months. For this reason, an effective predictive marker must be identified.

There has been much research evidence that the NLR is significantly related to the prognosis of CRC. The NLR is a potential prognostic and predictive factor for a variety of tumours, including CRC [24]. In the tumour microenvironment, genes encoding tumour-related macrophage activities, such as the LYZ (lysozyme), TYMP (thymidine phosphorylase), and CD68 (pan-macrophage) genes, may affect the NLR. TYMP promotes angiogenesis, escapes apoptosis, and stimulates tumour growth [25], and the accumulation of high levels of macrophages is positively correlated with high NLR levels. Neutrophils secrete a variety of proinflammatory, angiogenic and immunomodulatory factors, which have paracrine effects on tumour cell biology [26]. Lymphocytes induce cytotoxic cell death and produce cytokines that inhibit the proliferation and metastasis of cancer cells, thereby playing an important role in tumour suppression [27]. Neutrophils can inhibit the cytolytic activity of lymphocytes to tumour cells. In $\mathrm{CRC}$, an increase in the NLR value has an adverse effect on the survival rate of patients with CRC. However, this molecular mechanism is still complex and uncertain [28]. High NLR is associated with a highly aggressive tumour phenotype, and the degree of systemic inflammation is related to the subject's own physical state, which may weaken the subject's own tolerance and compliance, leading to reduced survival rates [29]. Our results show that the prognosis of subjects with NLR $<3.855$ is better than that of subjects with NLR $\geq 3.855$. Therefore, the NLR at baseline is an independent predictor of the OS of subjects treated with apatinib.

This study is a rigorously executed prospective study with strict ethical review. However, because onethird of the subjects in our clinical study had a baseline ECOG performance status score of 2 with simultaneous multisite metastases, the physical status of patients was relatively poor. Thus, the factors related to the poor prognosis of the subjects and biased enrolment led to unsatisfactory endpoints of OS and PFS in the study. In particular, the mismatch repair protein, RAS, RAF, and HER2 statuses were not standardized during enrolment. Accurate diagnosis has certain limitations. Our study is a single-arm design, and the small sample size affects the objectivity of our data. In the future, we will continue to carry out prospective randomized controlled clinical studies to include more subjects in strict accordance with the inclusion criteria, verify the efficacy and safety of apatinib combined with the FOLFIRI regimen, and further explore the relationship between the NLR and the curative effect of this treatment regimen.

\section{Conclusion}


This is a prospective, single-arm, single-centre, open phase II clinical study confirming that apatinib combined with the FOLFIRI regimen has a better curative effect and tolerance in patients with poor physical fitness and advanced unresectable CRC. It has an acceptable safety profile. It was verified that the baseline NLR can predict the efficacy of apatinib tablets combined with the FOLFIRI regimen.

Exploratory research was performed to further identify treatment options for advanced unresectable CRC.

\section{Declarations}

\section{Funding}

The work was supported by 2017 China Anti-Cancer Association- Hengrui Medicine Innovative Medicine Clinical Research Fund Project No.(CACA-CORP-143-027), The work was supported by Affiliated Cancer Hospital of Shanxi Medical University Project No.202006

\section{Conflict of interest}

The authors declare that there is no conflict of interest.

\section{Data avail ability statement}

The data that support the findings of this study are available from the corresponding authors upon reasonable request.

\section{Author Contributions}

Conceptualization: Haiyi Liu. Project administration and resource: Haiyi Liu,Yusheng Wang. Data curation: Xuetong Rong, Jie Wang, Jian Zhao. Data analysis: Xuetong Rong. Writing-original draft:Xuetong Rong. Statistical chart check: Hongmei Yu. Writing-review and editing: Yusheng Wang.

\section{Ethics statement}

This study is registered with ClinicalTrials.gov, number: ChiCTR1800015308. This study was performed in line with the princi-ples of the Declaration of Helsinki. Approval was granted by the Ethics Committee of Shanxi Cancer Hospital, and a signed informed consent was obtained from all the participants.

\section{Acknowledgments}

We thank the patients and their families/caregivers, the study investi-gators, and their staff.

\section{References}

1. Erratum: Global cancer statistics 2018: GLOBOCAN estimates of incidence and mortality worldwide for 36 cancers in 185 countries (2020). CA: a cancer journal for clinicians 70 (4):313. 
doi:10.3322/caac. 21609

2. Chen WQ, Li H, Sun KX, Zheng RS, Zhang SW, Zeng HM, Zou XN, Gu XY, He J (2018) [Report of Cancer Incidence and Mortality in China, 2014]. Zhonghua zhong liu za zhi [Chinese journal of oncology] 40 (1):5-13. doi:10.3760/cma.j.issn.0253-3766.2018.01.002

3. Modest DP, Pant S, Sartore-Bianchi A (2019) Treatment sequencing in metastatic colorectal cancer. European journal of cancer (Oxford, England : 1990) 109:70-83. doi:10.1016/j.ejca.2018.12.019

4. Tournigand C, André T, Achille E, Lledo G, Flesh M, Mery-Mignard D, Quinaux E, Couteau C, Buyse M, Ganem G, Landi B, Colin P, Louvet C, de Gramont A (2004) FOLFIRI followed by FOLFOX6 or the reverse sequence in advanced colorectal cancer: a randomized GERCOR study. Journal of clinical oncology : official journal of the American Society of Clinical Oncology 22 (2):229-237. doi:10.1200/jco.2004.05.113

5. Lyseng-Williamson KA (2012) Cetuximab: a guide to its use in combination with FOLFIRI in the firstline treatment of metastatic colorectal cancer in the USA. Molecular diagnosis \& therapy 16 (5):317322. doi:10.1007/s40291-012-0007-2

6. Venook AP, Niedzwiecki D, Lenz HJ, Innocenti F, Fruth B, Meyerhardt JA, Schrag D, Greene C, O'Neil BH, Atkins JN, Berry S, Polite BN, O'Reilly EM, Goldberg RM, Hochster HS, Schilsky RL, Bertagnolli MM, El-Khoueiry AB, Watson P, Benson AB, 3rd, Mulkerin DL, Mayer RJ, Blanke C (2017) Effect of First-Line Chemotherapy Combined With Cetuximab or Bevacizumab on Overall Survival in Patients With KRAS Wild-Type Advanced or Metastatic Colorectal Cancer: A Randomized Clinical Trial. Jama 317 (23):2392-2401. doi:10.1001/jama.2017.7105

7. van Helden EJ, Menke-van der Houven van Oordt CW, Heymans MW, Ket JCF, van den Oord R, Verheul HMW (2017) Optimal use of anti-EGFR monoclonal antibodies for patients with advanced colorectal cancer: a meta-analysis. Cancer metastasis reviews 36 (2):395-406. doi:10.1007/s10555-017-9668-y

8. Chen X, Qiu T, Zhu Y, Sun J, Li P, Wang B, Lin P, Cai X, Han X, Zhao F, Shu Y, Chang L, Jiang H, Gu Y (2019) A Single-Arm, Phase II Study of Apatinib in Refractory Metastatic Colorectal Cancer. The oncologist 24 (7):883-e407. doi:10.1634/theoncologist.2019-0164

9. Weitz J, Koch M, Debus J, Höhler T, Galle PR, Büchler MW (2005) Colorectal cancer. Lancet (London, England) 365 (9454):153-165. doi:10.1016/s0140-6736(05)17706-X

10. Onyoh EF, Hsu WF, Chang LC, Lee YC, Wu MS, Chiu HM (2019) The Rise of Colorectal Cancer in Asia: Epidemiology, Screening, and Management. Current gastroenterology reports 21 (8):36. doi:10.1007/s11894-019-0703-8

11. Zhang Y, Chen Z, Li J (2017) The current status of treatment for colorectal cancer in China: A systematic review. Medicine 96 (40):e8242. doi:10.1097/md.0000000000008242

12. Aziz MA, Serya RA, Lasheen DS, Abdel-Aziz AK, Esmat A, Mansour AM, Singab AN, Abouzid KA (2016) Discovery of Potent VEGFR-2 Inhibitors based on Furopyrimidine and Thienopyrimidne Scaffolds as Cancer Targeting Agents. Scientific reports 6:24460. doi:10.1038/srep24460

13. Li J, Zhao X, Chen L, Guo H, Lv F, Jia K, Yv K, Wang F, Li C, Qian J, Zheng C, Zuo Y (2010) Safety and pharmacokinetics of novel selective vascular endothelial growth factor receptor-2 inhibitor YN968D1 
in patients with advanced malignancies. BMC cancer 10:529. doi:10.1186/1471-2407-10-529

14. Cheng X, Feng H, Wu H, Jin Z, Shen X, Kuang J, Huo Z, Chen X, Gao H, Ye F, Ji X, Jing X, Zhang Y, Zhang T, Qiu W, Zhao R (2018) Targeting autophagy enhances apatinib-induced apoptosis via endoplasmic reticulum stress for human colorectal cancer. Cancer letters 431:105-114. doi:10.1016/j.canlet.2018.05.046

15. Lu W, Ke H, Qianshan D, Zhen W, Guoan X, Honggang Y (2017) Apatinib has anti-tumor effects and induces autophagy in colon cancer cells. Iranian journal of basic medical sciences 20 (9):990-995. doi:10.22038/ijbms.2017.9263

16. Cai X, Wei B, Li L, Chen X, Yang J, Li X, Jiang X, Lv M, Li M, Lin Y, Xu Q, Guo W, Gu Y (2020) Therapeutic Potential of Apatinib Against Colorectal Cancer by Inhibiting VEGFR2-Mediated Angiogenesis and $\beta$-Catenin Signaling. OncoTargets and therapy 13:11031-11044. doi:10.2147/ott.S266549

17. Liao X, Li H, Liu Z, Liao S, Li Q, Liang C, Huang Y, Xie M, Wei J, Li Y (2018) Clinical efficacy and safety of apatinib in patients with advanced colorectal cancer as the late-line treatment. Medicine 97 (50):e13635. doi:10.1097/md.0000000000013635

18. Takeuchi N, Koike K, Yoshida S, Kudo A, Sekiguchi N, Nakayama A, Kubota K, Rokuhara T, Kitahara M (2017) Treatment of metastatic refractory colorectal cancer following regorafenib failure. Molecular and clinical oncology 7 (2):308-312. doi:10.3892/mco.2017.1307

19. Li J, Qin S, Xu R, Yau TC, Ma B, Pan H, Xu J, Bai Y, Chi Y, Wang L, Yeh KH, Bi F, Cheng Y, Le AT, Lin JK, Liu T, Ma D, Kappeler C, Kalmus J, Kim TW (2015) Regorafenib plus best supportive care versus placebo plus best supportive care in Asian patients with previously treated metastatic colorectal cancer (CONCUR): a randomised, double-blind, placebo-controlled, phase 3 trial. The Lancet Oncology 16 (6):619-629. doi:10.1016/s1470-2045(15)70156-7

20. Carrato A, Benavides M, Massutí B, Ferreiro-Monteagudo R, García Alfonso P, Falcó E, Reboredo M, Cano T, Gallego J, Viéitez JM, Layos L, Salud A, Polo E, Dotor E, Durán-Ogalla G, Rodriguez-Garrote M, Calvo A, Grande E, Aranda E (2019) First-line single-agent regorafenib in frail patients with metastatic colorectal cancer: a pilot phase II study of the Spanish Cooperative Group for the Treatment of Digestive Tumours (TTD). BMC cancer 19 (1):533. doi:10.1186/s12885-019-5753-7

21. Zarour LR, Anand S, Billingsley KG, Bisson WH, Cercek A, Clarke MF, Coussens LM, Gast CE, Geltzeiler CB, Hansen L, Kelley KA, Lopez CD, Rana SR, Ruhl R, Tsikitis VL, Vaccaro GM, Wong MH, Mayo SC (2017) Colorectal Cancer Liver Metastasis: Evolving Paradigms and Future Directions. Cellular and molecular gastroenterology and hepatology 3 (2):163-173. doi:10.1016/j.jcmgh.2017.01.006

22. Yu X, Zhu L, Liu J, Xie M, Chen J, Li J (2020) Emerging Role of Immunotherapy for Colorectal Cancer with Liver Metastasis. OncoTargets and therapy 13:11645-11658. doi:10.2147/ott.S271955

23. Li J, Yuan Y, Yang F, Wang Y, Zhu X, Wang Z, Zheng S, Wan D, He J, Wang J, Ba Y, Bai C, Bai L, Bai W, Bi F, Cai K, Cai M, Cai S, Chen G, Chen K, Chen L, Chen P, Chi P, Dai G, Deng Y, Ding K, Fan Q, Fang W, Fang X, Feng F, Fu C, Fu Q, Gu Y, He Y, Jia B, Jiang K, Lai M, Lan P, Li E, Li D, Li J, Li L, Li M, Li S, Li Y, Li Y, Li Z, Liang X, Liang Z, Lin F, Lin G, Liu H, Liu J, Liu T, Liu Y, Pan H, Pan Z, Pei H, Qiu M, Qu X, Ren 
L, Shen Z, Sheng W, Song C, Song L, Sun J, Sun L, Sun Y, Tang Y, Tao M, Wang C, Wang H, Wang J, Wang S, Wang X, Wang X, Wang Z, Wu A, Wu N, Xia L, Xiao Y, Xing B, Xiong B, Xu J, Xu J, Xu N, Xu R, Xu Z, Yang Y, Yao H, Ye Y, Yu Y, Yu Y, Yue J, Zhang J, Zhang J, Zhang S, Zhang W, Zhang Y, Zhang Z, Zhang Z, Zhao L, Zhao R, Zhou F, Zhou J, Jin J, Gu J, Shen L (2019) Expert consensus on multidisciplinary therapy of colorectal cancer with lung metastases (2019 edition). Journal of hematology \& oncology 12 (1):16. doi:10.1186/s13045-019-0702-0

24. Wang F, Yuan X, Jia J, Bi X, Zhou Z, Zhou Q, Li X, Luo C, Deng M, Yi L, Li Y, Lu J, Su W, Chen H, Zhu Y, Wang S (2020) Apatinib Monotherapy for Chemotherapy-Refractory Metastatic Colorectal Cancer: A Multi-centre, Single-Arm, Prospective Study. Scientific reports 10 (1):6058. doi:10.1038/s41598-02062961-5

25. Sunakawa Y, Yang D, Cao S, Zhang W, Moran M, Astrow SH, Hsiang J, Stephens C, Tsuji A, Takahashi T, Tanioka H, Negoro Y, Takagane A, Tani S, Yamaguchi T, Eto T, Fujii M, Ichikawa W, Lenz HJ (2018) Immune-related Genes to Dominate Neutrophil-lymphocyte Ratio (NLR) Associated With Survival of Cetuximab Treatment in Metastatic Colorectal Cancer. Clinical colorectal cancer 17 (4):e741-e749. doi:10.1016/j.clcc.2018.08.002

26. Ogawa R, Yamamoto T, Hirai H, Hanada K, Kiyasu Y, Nishikawa G, Mizuno R, Inamoto S, Itatani Y, Sakai Y, Kawada K (2019) Loss of SMAD4 Promotes Colorectal Cancer Progression by Recruiting Tumor-Associated Neutrophils via the CXCL1/8-CXCR2 Axis. Clinical cancer research : an official journal of the American Association for Cancer Research 25 (9):2887-2899. doi:10.1158/10780432.Ccr-18-3684

27. Tan D, Fu Y, Tong W, Li F (2018) Prognostic significance of lymphocyte to monocyte ratio in colorectal cancer: A meta-analysis. International journal of surgery (London, England) 55:128-138. doi:10.1016/j.jjsu.2018.05.030

28. Li MX, Liu XM, Zhang XF, Zhang JF, Wang WL, Zhu Y, Dong J, Cheng JW, Liu ZW, Ma L, Lv Y (2014) Prognostic role of neutrophil-to-lymphocyte ratio in colorectal cancer: a systematic review and metaanalysis. International journal of cancer 134 (10):2403-2413. doi:10.1002/ijc.28536

29. Scott HR, McMillan DC, Forrest LM, Brown DJ, McArdle CS, Milroy R (2002) The systemic inflammatory response, weight loss, performance status and survival in patients with inoperable nonsmall cell lung cancer. British journal of cancer 87 (3):264-267. doi:10.1038/sj.bjc.6600466

\section{Figures}




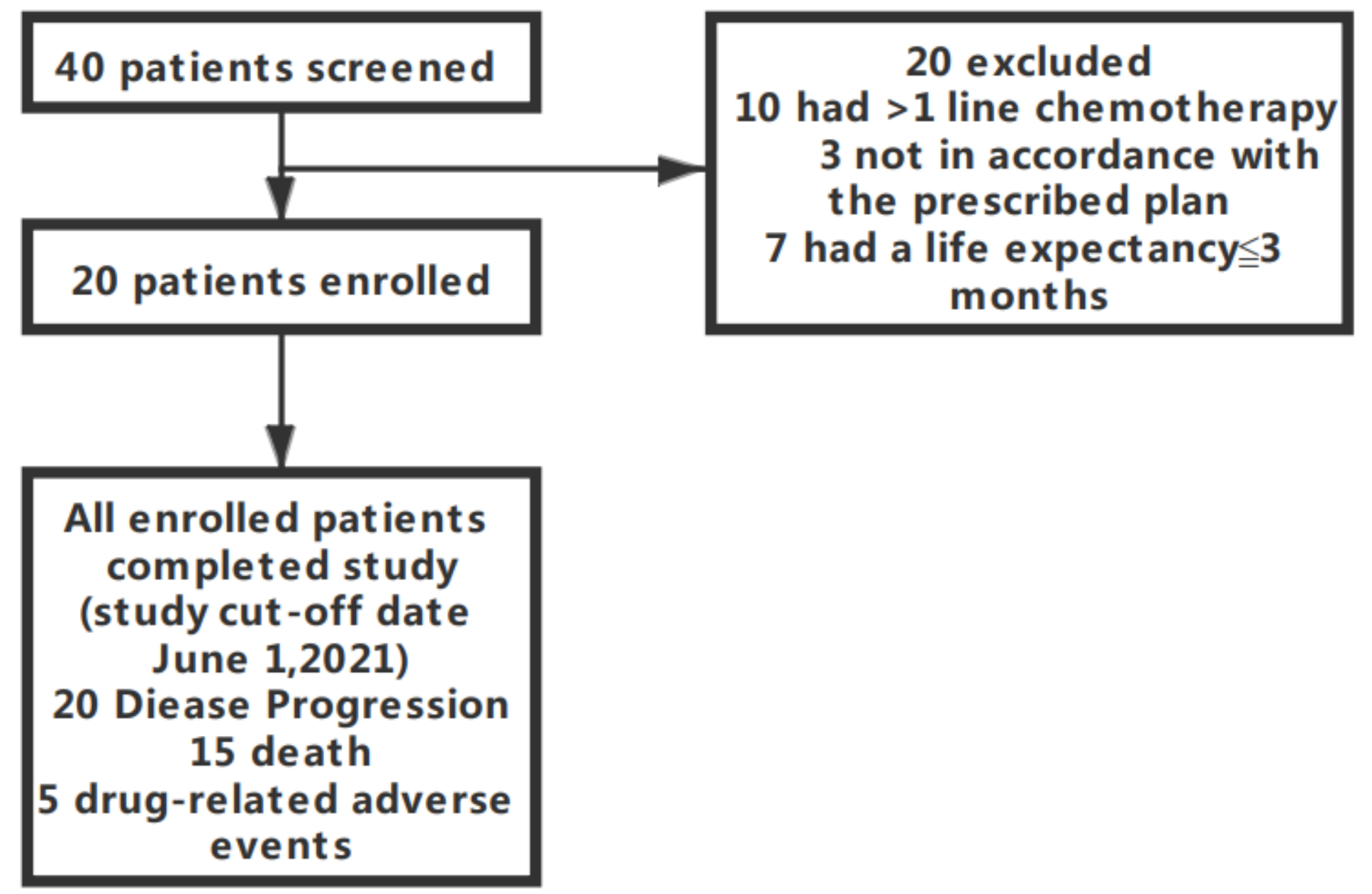

Figure 1

CONSORT diagram of study population selection for chemotherapy-refractory metastatic colorectal cancer 


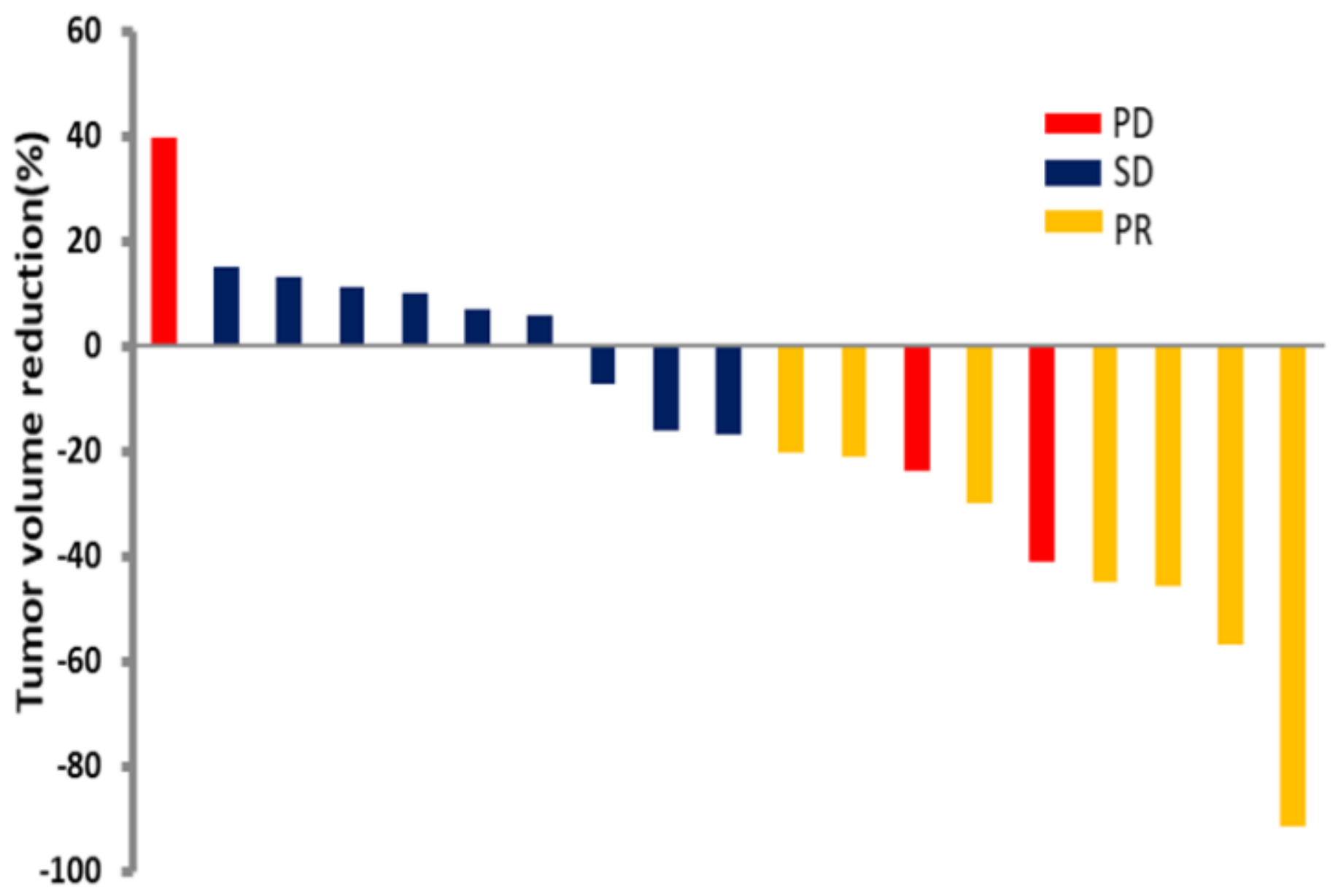

Figure 2

Waterfall plot of best percentage change from baseline in measurable tumour lesions

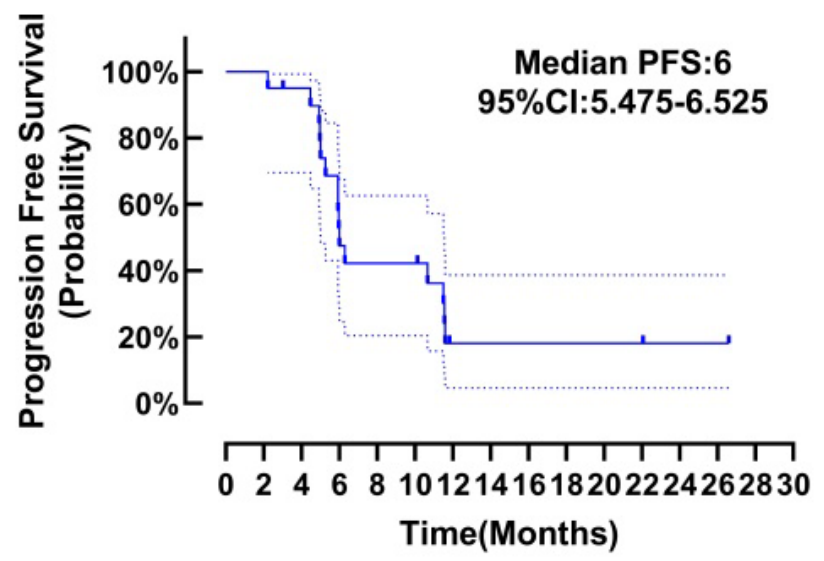

a

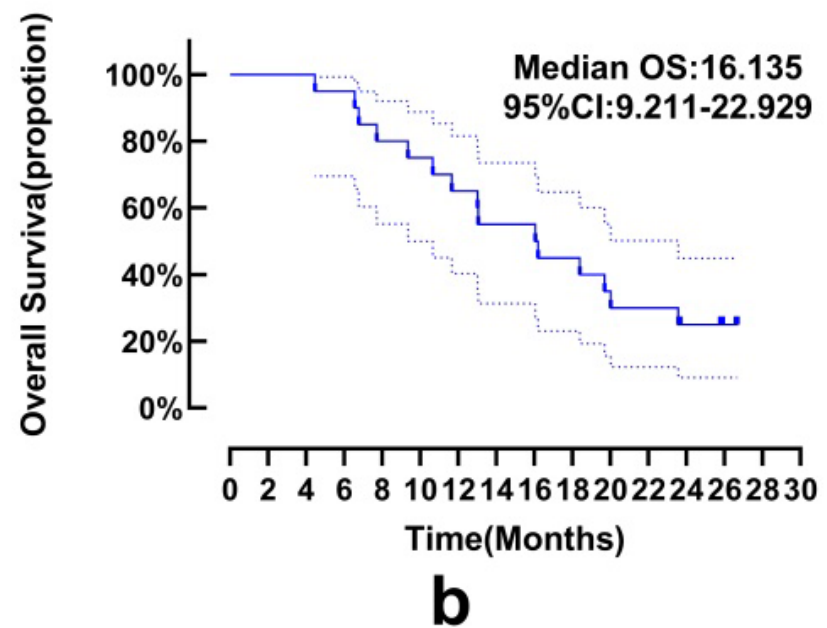

Figure 3

Kaplan-Meier curves for PFS (a) and OS(b) of the enrolled patients. PFS, progression-free survival; OS, overall survival; $\mathrm{Cl}$, confidence interval 
PFS

variables

Age $(<58.5 /=58.5$ )

Sex(male/female)

ECOG(1/2)

Primary(rectum)

Primary(left)

Primary(right)

Primary(sigmoid)

Differentiation

KRAS(yes)

KRAS(no)

KRAS(unknown)

Number of metastatic sites

Etastaticsite(Multi-site)

Etastaticsite(lung)

Etastaticsite(liver)

NLR

CA199

CEA

$\begin{array}{cc}H R(95 \% \mathrm{Cl}) & P \text { value } \\ 1.509(0.512-4.450) & 0.455 \\ 0.732(0.258-2.075) & 0.558 \\ 2.105(0.735-6.029) & 0.166 \\ & 0.192 \\ 1.046(0.126-8.661) & 0.967 \\ 2.585(0.723-9.237) & 0.144 \\ 0.226(0.028-1.811) & 0.161 \\ 2.445(0.810-7.378) & 0.113 \\ & 0.771 \\ 0.583(0.130-2.619) & 0.481 \\ 0.730(0.218-2.444) & 0.61 \\ 2.452(0.543-11.073) & 0.244 \\ & 0.405 \\ 0.261(0.033-2.062) & 0.203 \\ 0.641(0.142-2.896) & 0.563 \\ 2.350(0.840-6.568) & 0.103 \\ 1.383(0.470-4.065) & 0.556 \\ 3.202(0.842-12.176) & 0.088 \\ & \end{array}$

$\begin{array}{cc}\text { HR(95\%Cl) } & \text { P value } \\ 1.510(0.506-4.508) & 0.46 \\ 0.885(0.314-2.495) & 0.818 \\ 4.18(1.132-15.497) & 0.032 \\ & 0.62 \\ 0.907(0.113-7.303) & 0.927 \\ 1.307(0.399-4.276) & 0.658 \\ 0.294(0.037-2.332) & 0.246 \\ 2.150(0.751-6.152) & 0.154 \\ & 0.68 \\ 0.528(0.117-2.390) & 0.407 \\ 0.890(0.267-2.964) & 0.849 \\ 2.78(0.618-12.505) & 0.183 \\ & 0.392 \\ 0.309(0.040-2.414) & 0.263 \\ 0.496(0.109-2.244) & 0.362 \\ 3.851(1.33-11.152) & 0.013 \\ 1.106(0.376-3.249) & 0.855 \\ 3.826(0.931-15.716) & 0.063 \\ & \end{array}$

\section{Figure 4}

Univariate analysis of factors to predict progression-free survival and overall survival of apatinib shown by forest plot. PFS, progression-free survival; OS, overall survival; ECOG, Eastern Cooperative Oncology Group; NLR, neutrophil-to-lymphocyte ratio; CEA, carcinoembryonic antigen; CA19-9, carbohydrate antigen 19-9; HR, hazard ratio; $\mathrm{Cl}$, confidence interval; KRAS(yes), KRAS mutation; KRAS(no), no KRAS mutation; KRAS(unknown), unknown KRAS mutation status
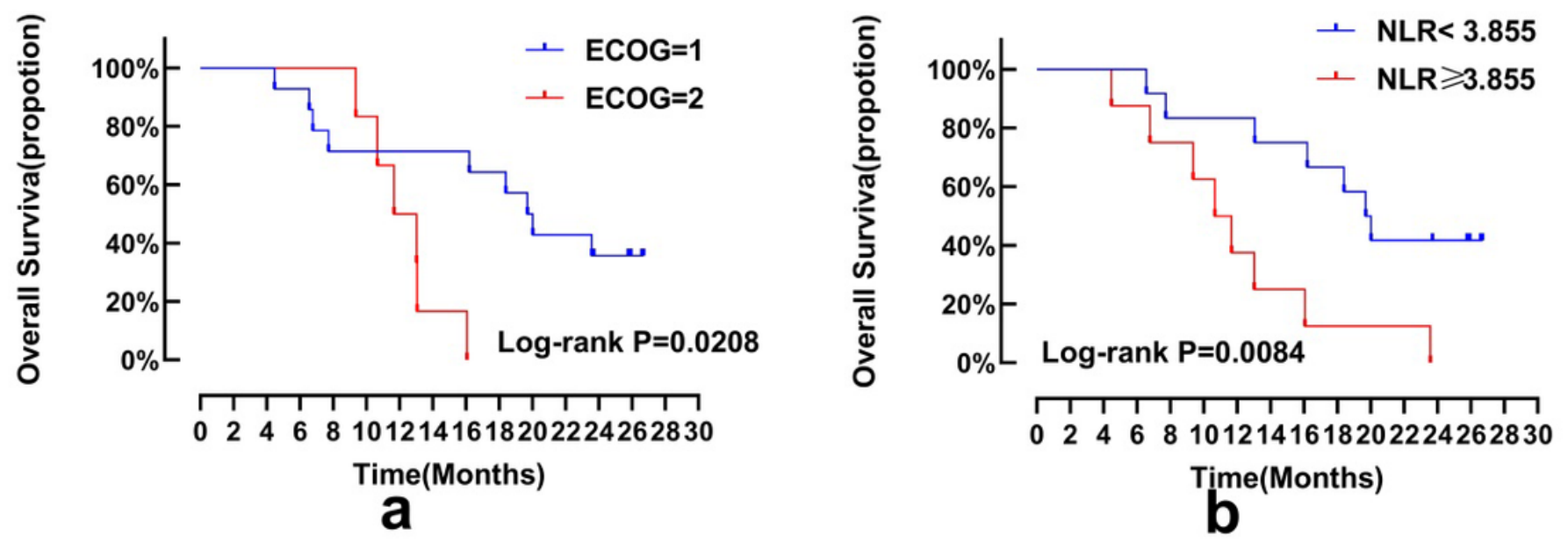

\section{Figure 5}

Kaplan-Meier estimates of subgroup analyses. Notes: (a) OS and (b) OS were estimated for patients with different ECOG and NLR levels. NLR, neutrophil-to-lymphocyte ratio; ECOG, Eastern Cooperative Oncology Group; OS, overall survival 